\title{
Severe water intoxication and secondary depressive syndrome in relation to delusional infestation
}

This article was published in the following Dove Press journal:

Neuropsychiatric Disease and Treatment

29 February 2016

Number of times this article has been viewed

\author{
Jianbo Lai' \\ Qiaoqiao Lu' \\ Yi Xu $u^{1,2}$ \\ Shaohua $\mathrm{Hu}^{1,2}$ \\ 'Department of Psychiatry, First \\ Affiliated Hospital, Zhejiang University \\ School of Medicine, Hangzhou, \\ People's Republic of China; ${ }^{2}$ The \\ Key Laboratory of Mental Disorder's \\ Management in Zhejiang Province, \\ Hangzhou, People's Republic of China
}

Correspondence: Shaohua $\mathrm{Hu}$

Department of Psychiatry, First Affiliated

Hospital, Zhejiang University School

of Medicine, No 79, Qingchun Road,

Hangzhou 310003, People's Republic

of China

Tel +86 57I 56723002

Fax $+8657 \mid 5672300$ I

Email dorhushaohua@zju.edu.cn

\begin{abstract}
This study presents a case of severe water intoxication in a female patient with delusional infestation. Self-induced excessive water ingestion is a rare medical condition, which has not been reported in patients with delusional infestation yet. The patient in this case study was a 60 -year-old Chinese woman, who was admitted to our hospital because of a feeling of skin infestation. She suffered from loss of consciousness and generalized tonic-clonic seizure after drinking $12 \mathrm{~L}$ of water during bowel cleansing before colonoscopy. Sufficient laboratory and imaging examinations were performed to exclude other possible causes of severe hyponatremia, such as hypothyroidism, diabetes insipidus, and syndrome of inappropriate antidiuretic hormone. Besides, the cystic lesion in the posterior pituitary revealed by cranial magnetic resonance imaging was not accountable for her delusional symptoms as well as excessive drinking behavior. Her delusional symptoms were in complete remission with a combination of risperidone and aripiprazole. However, nearly 3 months after discharge, this patient suffered from depressed mood and was diagnosed with depressive syndrome, and even attempted suicide. This case highlights the possibility of self-induced water intoxication in patients with delusional infestation, inevitably adding to the complexity of the disease, and indicates the necessity of precautions for secondary psychotic or mood problems after symptomatological remission.
\end{abstract}

Keywords: delusional infestation, depressive syndrome, suicide, water intoxication

\section{Introduction}

Delusional infestation (DI) is a rare psychocutaneous condition complicated by various causes and comorbidities. In contrast to the primary form of DI, which is independent from underlying diseases, the secondary form of DI presents as a concomitant phenomenon in other dominant conditions. ${ }^{1}$ As was documented in literature, neuropsychiatric diseases, psychotropic drugs, nutrient deficiency, infections, and even homeostatic imbalance all possibly facilitate the onset and development of secondary DI. ${ }^{2}$ Selfinduced water intoxication is another uncommon emergency condition that needs prompt management. However, it has been reported that exaggerated water intake may occur in almost any psychiatric disorder, especially those with psychotic traits. ${ }^{3}$ Self-induced water intoxication in patients with DI has never been previously documented.

In affected patients, DI significantly weakens psychosocial functioning and impairs the quality of life. Owing to the lack of insight on disease nature, patients with DI are always reluctant to visit a psychiatrist and have a poor compliance, which further worsens their condition. These patients easily feel anxious, and as a result, establishing a therapeutic rapport with them requires great effort. Managing primary diseases is the immediate task, and antipsychotics can be used to eliminate psychotic symptoms 
related to DI. ${ }^{2,4}$ However, long-term follow-ups were not found to be of great importance in previous studies after a full remission from DI.

This study reports an impressive case of a female patient with DI who developed generalized tonic-clonic seizure after drinking $12 \mathrm{~L}$ of water during bowel cleansing before colonoscopy. After recovering from acute water intoxication, her delusional symptoms were well alleviated by a combination of risperidone and aripiprazole. However, nearly 3 months after discharge, this patient suffered from depressed mood and was diagnosed with depressive syndrome. Her mood problem did not respond well to the antidepressant therapy, and she even made a suicide attempt. This single case highlighted that patients with DI may also have a risk of self-induced excessive water intake, which inevitably adds to the complexity of the disease and warranted the importance of continuous health monitoring for possible psychotic or mood disorders during follow-ups. This work was approved by the First Affiliated Hospital of Zhejiang University School of Medicine Ethics Committee. Written informed consent was given by the patient after her recovery from water intoxication.

\section{Case presentation}

Mrs X, a 60-year-old Chinese woman, was admitted to the Department of Infectious Diseases, First Affiliated Hospital of Zhejiang University School of Medicine, because of the feeling of skin infestation. Since her travel to a rural area 4 months ago, she had been persistently feeling insects crawling and breeding under her skin. She complained that these insects bit her skin, causing intolerable pain and itch. She insisted that her living room was full of insects of different sizes, and numerous insect eggs could have washed off when washing her hair. Before admission, she was diagnosed with "pediculosis, allergic dermatitis", and prescribed desloratadine and metronidazole by a dermatologist. Not surprisingly, these agents did not help relieve her sufferings. Since the onset of the aforementioned symptoms, her body weight consequently went down by $8 \mathrm{~kg}$.

In her past medical history, she suffered from hypertension for 9 years, which is well controlled by amlodipine $5 \mathrm{mg}$ daily. She denied any personal history of other physical or mental illnesses, illicit drug abuse, or intoxication, as well as a family history of psychiatric conditions. No irritant or traumatic events happened before her current presentation. Besides, she was allergic to sulfonamides and levofloxacin. She had an open appendectomy 40 years before admission and gave birth to her son by cesarean delivery 30 years before admission.

A full-body skin examination revealed scattered excoriations with pigmentation caused by self-inflicted scratching. Further laboratory screenings, including profiles of routine blood, urine, stool tests, serum biochemistry, coagulation function, thyroid function, myocardial enzymes, troponin-I, C-reactive protein, rheumatoid factor, erythrocyte sedimentation rate, serum tumor markers, antinuclear antibodies, prolactin, serum VitB12, folate level, hepatitis B and C, human immunodeficiency virus, syphilis, cytomegalovirus, and Epstein-Barr virus, were all within normal limits except that fecal occult blood test was positive. Besides, electrocardiogram and chest computed tomography (CT) were normal. Color Doppler ultrasonography detected a diffusely enlarged thyroid with multiple nodules, and a small hepatic cyst. Cranial magnetic resonance imaging (MRI) (3.0 T) indicated a cystic lesion in the posterior pituitary (Figure 1A).
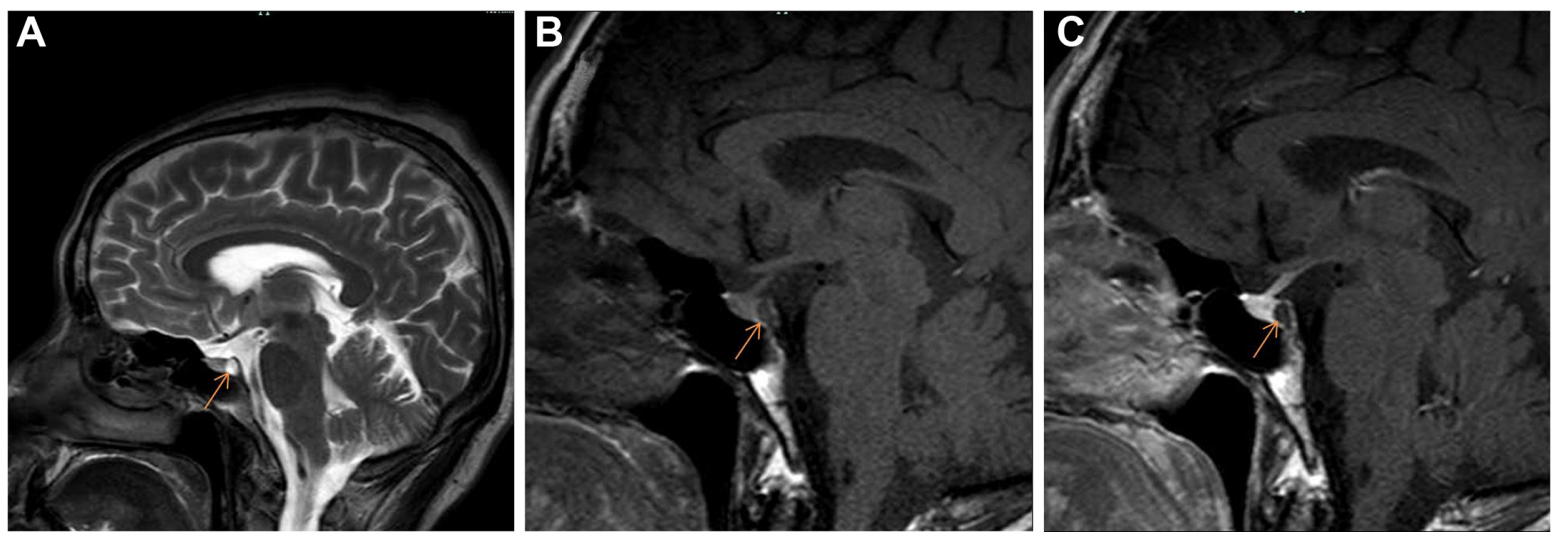

Figure I Data from cranial 3.0 T MRI scanning.

Notes: (A) A cystic lesion (orange arrow) in the posterior pituitary with high signal intensity on T2 weight sequence (data collected before water intoxication); (B) low signal intensity of the cystic lesion on TI weight sequence (data collected after prolactin elevation); (C) following the injection of contrast, signal intensity in the cystic lesion was not obviously enhanced on TI weight sequence (data collected after prolactin elevation).

Abbreviation: MRI, magnetic resonance imaging. 
Meanwhile, the patient complained about archorrhagia and insect eggs washing off when cleaning her anus. Therefore, an anorectal consultation was immediately requested. Digital rectal examination was negative, and colonoscopy was suggested. Unexpectedly, when preparing for a bowel cleansing before colonoscopy, this patient ingested five thermos jugs of water (appropriately $12 \mathrm{~L}$ ) in a few hours. Consequently, she began to feel short of breath, although bedside heart and lung auscultation was normal. Blood pressure was 143/76 mmHg. About 20 minutes later, she became irritated and could not stop scratching her face and head. Rapid blood glucose was $7.7 \mathrm{mmol} / \mathrm{L}$. After another half an hour, in a sitting position, the patient vomited appropriately $50 \mathrm{~mL}$ of water, with eyes tightly closed and hands involuntarily trembling. Direct and consensual reactions on both pupils were sensitive. Her neck was soft, but bilateral Babinski's sign became positive. Another 15 minutes later, the patient experienced a seizure, characterized by loss of consciousness, turning up of the eyes, biting of the tongue, clenching of the teeth, frothing of the mouth, and urinary incontinence. After about 5 minutes, seizure gradually alleviated, but the patient remained irritated and her hands repetitively grasped in an involuntary pattern. Immediately, $10 \mathrm{mg}$ of diazepam was intramuscularly injected. A neurological consult considered the aforementioned symptoms to be an epileptic attack and suggested the use of sodium valproate. A quick check on serum electrolytes indicated hyponatremia $(120 \mathrm{mmol} / \mathrm{L}$, reference interval: 136-145 mmol/L), hypochloremia ( $82 \mathrm{mmol} / \mathrm{L}$, reference interval: $96-108 \mathrm{mmol} / \mathrm{L})$, and hypokalemia $(3.27 \mathrm{mmol} / \mathrm{L}$, reference interval: $3.50-5.20 \mathrm{mmol} / \mathrm{L}$ ), which possibly linked to the overintake of water and accounted for the epilepticus insultus. Cranial CT acquisitions were generally normal, although data were not that clear because of noncooperation of the patient. The results of lumbar puncture excluded intracranial infections and revealed a low level of cerebrospinal fluid chlorine. The arterial blood gas analysis indicated uncompensated alkalosis ( $\mathrm{pH}$ value 7.52, reference interval: 7.35-7.45). As the situation was urgent, the patient was transferred to the Department of Intensive Care Unit (ICU) for further examinations and treatments. In the ICU, vital signs and urine volume of this patient were closely monitored. She was treated with meticulous supportive and nursing care (eg, oxygen uptake, catheter care, and perineal cleaning), intravenous sodium valproate pumping, nasal levetiracetam feeding, and intravenous potassium and sodium supplement at a well-controlled speed. The patient has experienced fever when she was in a mild coma about
1 day after the epileptic attack, and her body temperature increased to a maximum of $39^{\circ} \mathrm{C}$. The auscultation of lungs revealed bilateral crackles, and the routine blood test revealed increased count of white blood cells and neutrophils over normal limits. Lung infection was therefore indicated, and antibiotics were prescribed. Meanwhile, hematological tests documented elevated hepatic enzymes and cardiac enzymes. In addition, urine sodium and osmolality, and serum adrenocorticotropic hormone and cortisol levels were screened to exclude diabetes insipidus and syndrome of inappropriate antidiuretic hormone (SIADH). About 45 hours after the epileptic attack, her deteriorated consciousness completely cleared, whereas her cutaneous concerns remained the same as before. After 4 days in the ICU, laboratory examinations basically returned to normal, and the patient was transferred back to the Department of Infectious Diseases. Psychiatric consulting was asked to deal with her persistent psychotic symptoms. Because the patient had abnormal skin sensations without any trace of infection, confirmedly convincing herself of being infected with insects, she met the criteria proposed in $2009^{2}$ and was thereof diagnosed with DI. Accordingly, she was administered risperidone, titrated to $4 \mathrm{mg} /$ day (1.5 $\mathrm{mg}$ at noon and $2.5 \mathrm{mg}$ at night). However, laboratory monitoring revealed an elevated serum level of prolactin (highest at $189.6 \mathrm{ng} / \mathrm{mL}$, reference interval: 2.8-29.2 mmol/L). Furthermore, 3.0 T MRI scanning of the pituitary reported a similar result as earlier (Figure 1B and $\mathrm{C}$ ). Considering risperidone to be the culprit for prolactin disturbance, its dose was gradually decreased to $2.5 \mathrm{mg} / \mathrm{d}$ (night only), and $5 \mathrm{mg}$ aripiprazole (night only) was added together with bromocriptine. Consequently, serum prolactin gradually declined to the normal level. After a 2-month inpatient treatment with atypical antipsychotics, her tactile hallucinations and somatic delusions significantly improved, and continuous medication was suggested after discharge.

During outpatient follow-ups, symptomatological remission of DI was maintained. However, the patient developed symptoms of depressed mood and loss of appetite and interest nearly 3 months after hospital discharge. In a psychiatric visit, her Hamilton Depression Rating Scale (17 items) reached a score of 27 . She was diagnosed with depressive syndrome after comprehensive psychiatric assessments. Accordingly, citalopram was prescribed and gradually titrated to $40 \mathrm{mg} /$ day. Meanwhile, she was advised to continue her antipsychotics for DI. However, her depressed mood did not significantly improve after a 2 -week treatment with citalopram. Nearly 1 month after citalopram was initiated, this woman was reported to have attempted suicide in her 
own house. She was found trying to jump from the balcony and was stopped after much persuasion. She was then inevitably lost to follow-up, although repeated phone calls were made.

\section{Discussion}

Severe water intoxication elicited by drinking excessive water is a rare emergency condition. The overintake of water causes a rapid decline in serum electrolyte concentration and encephaledema, which further disrupts neuron membrane stability and even progresses to seizure in some susceptible individuals. ${ }^{5}$ In this case with DI, the patient unexpectedly ingested a large amount of water during bowel cleansing before colonoscopy. The overconsumption of approximately $12 \mathrm{~L}$ of water in a few hours was indeed a terrifying thing, which ultimately caused a grand mal seizure in this patient. Sufficient laboratory and imaging examinations were performed to exclude other possible causes of severe hyponatremia, such as hypothyroidism, diabetes insipidus, and SIADH. Besides, the cystic lesion in the posterior pituitary revealed by cranial MRI was not accountable for her delusional symptoms as well as excessive drinking behavior. Before hospitalization, medications for "pediculosis" were of poor efficacy. However, a combination of risperidone and aripiprazole helped to dispel her cutaneous concerns. A final diagnosis of DI was further confirmed.

Still, this incident that happened in a hospital complicated the course of disease and was worth reflecting on. Whether this patient had a chronic overintake of water before admission was unknown; however, bowel cleansing before colonoscopy indeed became a prerequisite for her dangerous excessive water ingestion. Considering patients with DI always being in a state of anxiety and lacking insights into disease nature, clinicians are responsible to make patients and their caregivers understand every detail of any examination. Besides, previous studies have reported that patients with psychiatric disorders had an increased risk for self-induced water intoxication, ${ }^{3}$ which was known as psychogenic polydipsia. ${ }^{6}$ It is estimated that psychogenic polydipsia has an incidence of approximately $6 \%-20 \%$ in psychiatric patients. ${ }^{7}$ Chronic schizophrenics, chronic alcoholics, and middle-aged women with anxiety disorders constitute the main part of this population. ${ }^{3,8,9}$ For example, one case report documented a schizophrenic patient who drank a large quantity of water to flush away imaginary parasites from his body and consequently suffered a similar course of acute water intoxication as observed in the present study. ${ }^{10}$ Therefore, it is reasonable to speculate that the patient in the present case drank excessive water possibly for the same reason. Such drinking behavior, together with self-inflicted skin scratches, reflected a lack of insight into the disease nature in these patients, which may also be used to assess the severity of symptoms. In addition, psychotic and anxious traits in patients with DI may also contribute to their compulsive water drinking.

In outpatient follow-ups, considering that medication adjustment to monotherapy might weaken her compliance, this patient was advised to continue her antipsychotics. She was still taking risperidone and aripiprazole when citalopram was added for depression treatment. It has already been reported that severe secondary depression occurred in more than half of DI patients, and suicide ideations or attempts were not uncommon, ${ }^{11}$ which was further confirmed in the present case. In a recent structural neuroimaging study, alterations in prefrontal and temporal areas were indicated in patients with DI, ${ }^{12}$ which were consistent with the findings in patients with major depressive disorder. ${ }^{13}$ Therefore, DI and depression may not be two isolated psychiatric conditions. They seem to present as different manifestations or stages of a disease spectrum, which means these two conditions possibly share intimate neural correlates.

To conclude, although underlying neural pathophysiology of DI remains largely unknown, the clinical picture of the patient in this case highlights that patients with DI may have a potential risk for self-induced water intoxication, which deserves more attention in clinical practice. Moreover, this case emphasizes that long-term follow-ups are necessary even after symptom remission to monitor the possible onset of other psychotic or mood disorders in later life.

\section{Disclosure}

The authors report no conflicts of interest in this work.

\section{References}

1. Ganner H, Lorenzi E. Delusions of skin infestations. Psychiatr Clin (Basel). 1975;8(1-2):31-44.

2. Freudenmann RW, Lepping P. Delusional infestation. Clin Microbiol Rev. 2009;22(4):690-732.

3. Ferrier IN. Water intoxication in patients with psychiatric illness. Br Med J. 1985;291(6509):1594-1596.

4. Heller MM, Wong JW, Lee ES, et al. Delusional infestations: clinical presentation, diagnosis and treatment. Int J Dermatol. 2013;52(7): 775-783.

5. Fraser CL, Arieff AI. Epidemiology, pathophysiology, and management of hyponatremic encephalopathy. Am J Med. 1997;102(1):67-77.

6. Illowsky BP, Kirch DG. Polydipsia and hyponatremia in psychiatric patients. Am J Psychiatry. 1988;145(6):675-683.

7. Verghese C, de Leon J, Josiassen RC. Problems and progress in the diagnosis and treatment of polydipsia and hyponatremia. Schizophr Bull. 1996;22(3):455-464.

8. Dundas B, Harris M, Narasimhan M. Psychogenic polydipsia review: etiology, differential, and treatment. Curr Psychiatry Rep. 2007;9(3): 236-241. 
9. Cronin RE. Psychogenic polydipsia with hyponatremia: report of eleven cases. Am J Kidney Dis. 1987;9(5):410-416.

10. Alexander ER, Crow TJ, Hamilton SM. Water intoxication in relation to acute psychotic disorder. Br Med J. 1973;1(5845):89.

11. Munro A. Monosymptomatic hypochondriacal psychosis. Br J Psychiatry Suppl. 1988;(2):37-40.
12. Wolf RC, Huber M, Depping MS, et al. Abnormal gray and white matter volume in delusional infestation. Prog Neuropsychopharmacol Biol Psychiatry. 2013;46:19-24.

13. Fung G, Deng Y, Zhao Q, et al. Distinguishing bipolar and major depressive disorders by brain structural morphometry: a pilot study. BMC Psychiatry. 2015;15(1):298.

\section{Publish your work in this journal}

Neuropsychiatric Disease and Treatment is an international, peerreviewed journal of clinical therapeutics and pharmacology focusing on concise rapid reporting of clinical or pre-clinical studies on a range of neuropsychiatric and neurological disorders. This journal is indexed on PubMed Central, the 'PsycINFO' database and CAS, and is the official journal of The International Neuropsychiatric Association (INA). The manuscript management system is completely online and includes a very quick and fair peer-review system, which is all easy to use. Visit http://www.dovepress.com/testimonials.php to read real quotes from published authors.

Submit your manuscript here: http://www.dovepress.com/neuropsychiatric-disease-and-treatment-journal 